ACCEPTED MANUSCRIPT

\title{
Graphene and graphene nanomesh supported nickel clusters: Electronic, magnetic, and hydrogen storage properties
}

To cite this article before publication: M M Fadlallah et al 2018 Nanotechnology in press https://doi.org/10.1088/1361-6528/aaee3c

\section{Manuscript version: Accepted Manuscript}

Accepted Manuscript is "the version of the article accepted for publication including all changes made as a result of the peer review process, and which may also include the addition to the article by IOP Publishing of a header, an article ID, a cover sheet and/or an 'Accepted

Manuscript' watermark, but excluding any other editing, typesetting or other changes made by IOP Publishing and/or its licensors"

This Accepted Manuscript is @ 2018 IOP Publishing Ltd.

During the embargo period (the 12 month period from the publication of the Version of Record of this article), the Accepted Manuscript is fully protected by copyright and cannot be reused or reposted elsewhere.

As the Version of Record of this article is going to be / has been published on a subscription basis, this Accepted Manuscript is available for reuse under a CC BY-NC-ND 3.0 licence after the 12 month embargo period.

After the embargo period, everyone is permitted to use copy and redistribute this article for non-commercial purposes only, provided that they adhere to all the terms of the licence https://creativecommons.org/licences/by-nc-nd/3.0

Although reasonable endeavours have been taken to obtain all necessary permissions from third parties to include their copyrighted content within this article, their full citation and copyright line may not be present in this Accepted Manuscript version. Before using any content from this article, please refer to the Version of Record on IOPscience once published for full citation and copyright details, as permissions will likely be required. All third party content is fully copyright protected, unless specifically stated otherwise in the figure caption in the Version of Record.

View the article online for updates and enhancements. 


\title{
Graphene and graphene nanomesh supported nickel
}

clusters: Electronic, magnetic, and hydrogen storage

\author{
properties

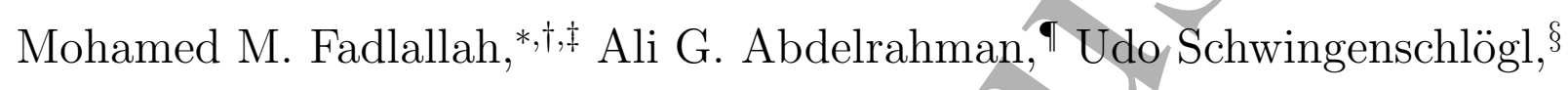 \\ and Ahmed A. Maarouf*,\| \\ $\nmid$ †epartment of Physics, Faculty of Science, Benha University, Benha, Egypt \\ †Center for Nanotechnology, Zewail City of Science and Technology, Giza 12588, Egypt \\ ФDepartment of Basic Science, Faculty of Computer and Information Science, Ain Shams \\ University, Cairo, Egypt \\ $\S$ King Abdullah University of Science and Technology (KAUST), Physical Science and \\ Engineering Division (PSE), Thuwal 23955-6900, Saudi Arabia \\ \|Department of Physics, Institute for Research and Medical Consultations, Imam \\ Abdulrahman Bin Faisal University, Dammam 31441, Saudi Arabia \\ E-mail: mohamed.fadlallah@fsc.bu.edu.eg; amaarouf@iau.edu.sa
}

November 1, 2018

\section{Abstract}

Small-sized nanoparticles are widely used in applications such as catalysis, nanoelectronics, and hydrogen storage. However, the small size causes a common problem: agglomeration on the support template. One solution is to use templates that limit the mobility of the nanoparticles. Graphene nanomeshes (GNMs) are two dimensional 
porous structures with controllably passivated pores. In this work, we employ first principles calculations to investigate the potential for using GNMs as support templates for $\mathrm{Ni}$ clusters and, at the same time, study their magnetic and hydrogen storage properties. We consider two Ni clusters $\left(\mathrm{Ni}_{6}\right.$ and $\left.\mathrm{Ni}_{13}\right)$ and two GNMs (O-terminated and N-terminated), comparing our results to those of isolated Ni clusters and those of Ni clusters on graphene. High stability of the Ni clusters is found on the N-GNM in contrast to the O-GNM. We quantify the hydrogen storage capacity by calculating the adsorption energy for multiple $\mathrm{H}_{2}$ molecules. The values on $\mathrm{Ni}_{x} / \mathrm{N}-\mathrm{GNM}$ are significantly reduced as compared to the corresponding isolated $\mathrm{Ni}_{x}$ clusters, but a high hydrogen storage capacity is maintained. The fact that $\mathrm{Ni}_{x} / \mathrm{N}-\mathrm{GNM}$ hosts spin polarization is interesting for spintronic applications.

\section{Keywords}

Density functional theory, hydrogen storage, Ni cluster, graphene, nanomesh

\section{Introduction}

Carbon dioxide emissions from ordinary fuels and the consequent global warming are driving the quest for clean, renewable, and cheap energy sources. ${ }^{1}$ Hydrogen is one potential candidate, as it is environmentally friendly and has the highest energy per mass of any fuel. ${ }^{2}$ Therefore, high-capacity hydrogen storage is a desirable technological goal and many experimental $^{3,4}$ and theoretical ${ }^{3,5}$ efforts have aimed to improve the process. In addition to the gas/liquid storage of hydrogen in high-pressure vessels, a focus is developing on materialsbased storage. ${ }^{6,7}$ Due to their large surface area and moderate cost, C-based nanomaterials are considered for hydrogen storage. ${ }^{8}$ For example, carbon nanotubes doped with $\mathrm{Ca}, \mathrm{Co}, \mathrm{Fe}$, $\mathrm{Ni}$, and $\mathrm{Pd}$ have high storage capacities at standard temperature and pressure. ${ }^{9}$ Li trapped in a graphene double vacancy ${ }^{10}$ and $\mathrm{Ni}$-decorated graphene ${ }^{11}$ store up to $5 \mathrm{H}_{2}$ molecules per 
Li/Ni atom. High hydrogen storage capacities also are predicted for Ca-decorated zigzag graphene nanoribbons, ${ }^{12}$ porous graphene oxide, ${ }^{13}$ and porous graphene doped with various elements. ${ }^{14,15}$

A graphene nanomesh (GNM, also called porous graphene) is a two dimensional material formed by creating a lattice of pores in graphene. The sizes and geometries of the pores control the electronic properties of the GNM, which can be semiconducting or semimetallic. ${ }^{16-19}$ Pore passivation by species such as $\mathrm{H}, \mathrm{O}$, and $\mathrm{N}$ can be used to dope GNMs ${ }^{18,20,21}$ and control their magnetic properties. ${ }^{22}$ The pores also offer a possible route to boost the chemical reactivity, allowing GNMs to be utilized as templates in various applications, for example, as electrostatic and chemical traps. ${ }^{23}$ GNMs have been fabricated with pore sizes between $5 \mathrm{~nm}$ and $200 \mathrm{~nm},{ }^{24,25}$ making them suitable templates for catalytíc nanoparticles, ${ }^{11,23}$ molecular sensors, ${ }^{26,27}$ and nanoparticles for hydrogen storage. ${ }^{15}$

In this work, we consider $\mathrm{Ni}_{6}$ and $\mathrm{Ni}_{13}$ clusters, isolated, on graphene, and on $\mathrm{O}$ - and N-passivated GNMs (O-GNM and N-GNM, respectively). We study their magnetic and hydrogen storage properties both with and without templates.

\section{Computational methods}

All calculations are performed using the QUANTUM ESPRESSO plane wave density functional theory package. ${ }^{28}$ Spin-polarized calculations are employed to determine the electronic and magnetic properties. For the exchange correlation functional we use the generalized gradient approximation in the scheme of Perdew-Burke-Ernzerhof. A $9 \times 9 \times 1$ Monkhorst-Pack $^{29}$ $k$-mesh and a 40 Ry energy cut-off are used. A $6 \times 6$ graphene supercell is built, with a 15 $\AA$ thick vacuum separation to prevent interaction along the c-direction. GNMs with hexagonal pores are obtained by removing $12 \mathrm{C}$ atoms and saturating the pore edge with $6 \mathrm{O}$ or $6 \mathrm{~N}$ atoms. All systems are structurally relaxed to reduce the atomic forces below 0.002 $\mathrm{Ry} /$ Bohr. At the start of the structural relaxation the bottom of the Ni clusters is located 
about $3 \AA$ above the graphene/GNM plane.

\section{Results and discussion}

\section{$3.1 \mathrm{Ni}_{6}$ and $\mathrm{Ni}_{13}$ clusters}

We begin by studying the magnetic and hydrogen storage properties of $\mathrm{Ni}_{6}$ and $\mathrm{Ni}_{13}$ clusters, which are found experimentally ${ }^{30}$ and theoretically ${ }^{31}$ to be the most stable Ni clusters. Their optimized structures (Fig. 1a,b) show average Ni-Ni bond lengths of $2.31 \AA$ and $2.37 \AA$, respectively, and magnetic moments per Ni atom of $1.4 \mu_{B}$ and $0.9 \mu_{B}$, in agreement with previous calculations. ${ }^{31,32}$ The density of states (DOS) (Fig. 1c,d) demonstrates that the Fermi energy is located in the spin-down $\mathrm{Ni} 3 \mathrm{~d}$ states for $\mathrm{Ni}_{6}$ with a spin-up gap of about 2 $\mathrm{eV}$, while for $\mathrm{Ni}_{13}$ both spin channels are metallic. We note that all atoms of $\mathrm{Ni}_{6}$, but only 12 atoms of $\mathrm{Ni}_{13}$, constitute the surface of the cluster. 

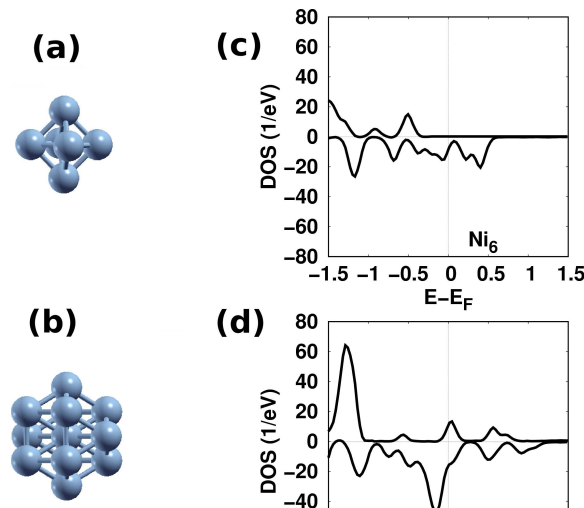

(d)
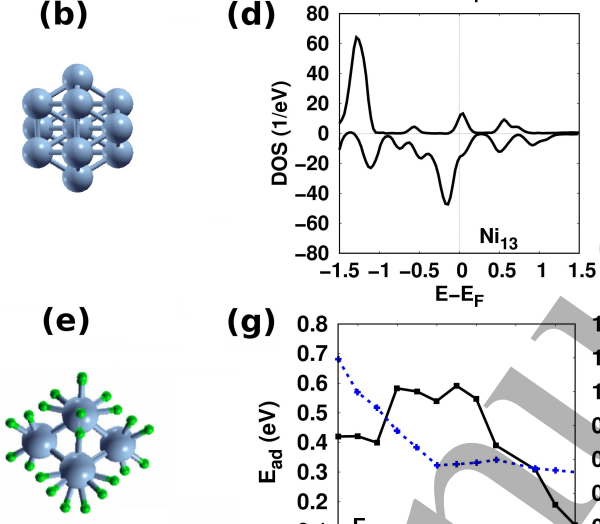

(g) 0.8
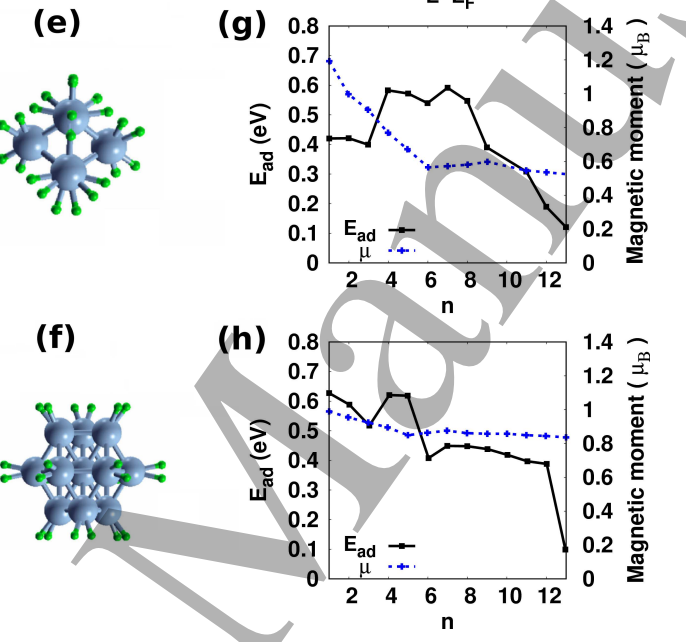

Figure 1: Optimized structures of (a) $\mathrm{Ni}_{6}$ and (b) $\mathrm{Ni}_{13}$, and (c,d) corresponding DOS. Optimized structures of (e) $\mathrm{Ni}_{6}$ and (f) $\mathrm{Ni}_{13}$ with $\mathrm{H}_{2}$ molecules adsorbed, and corresponding (g) $E_{\text {ad }}$ and (h) $\mu$ per $\mathrm{Ni}$ atom as functions of $n$.

We calculate the storage capacities of the $\mathrm{Ni}_{x}$ clusters $(x=6,13)$ by adding $\mathrm{H}_{2}$ molecules, one at a time,allowing the system to structurally relax after each addition. The adsorption energy of the $n^{\text {th }}$ molecule is

$$
E_{\mathrm{ad}}\left(\mathrm{H}_{2}^{(n)}\right)=E\left(\mathrm{Ni}_{x}+(n-1) \mathrm{H}_{2}\right)+E\left(\mathrm{H}_{2}\right)-E\left(\mathrm{Ni}_{x}+n \mathrm{H}_{2}\right)
$$

where $E\left(\mathrm{Ni}_{x}+n \mathrm{H}_{2}\right)$ is the total energy of the cluster with $n$ adsorbed $\mathrm{H}_{2}$ molecules and $E_{\mathrm{H}_{2}}$ is the total energy of an isolated $\mathrm{H}_{2}$ molecule. The isolated $\mathrm{Ni}_{6}$ and $\mathrm{Ni}_{13}$ clusters are capable of adsorbing up to $13 \mathrm{H}_{2}$ molecules with $E_{\text {ad }}$ larger than $0.1 \mathrm{eV}$ (Fig. 1e,f). For both 
clusters we find that the average $\mathrm{Ni}-\mathrm{Ni}$ bond length does not change after $\mathrm{H}_{2}$ adsorption, while the average $\mathrm{Ni}-\mathrm{H}$ bond length is $1.61 \AA$. The average $E_{\text {ad }}$ is $0.36 \mathrm{eV}$ for $\mathrm{Ni}_{6}$ and 0.41 $\mathrm{eV}$ for $\mathrm{Ni}_{13}$.

The magnetic moments of both clusters tend to decrease with increasing number of adsorbed $\mathrm{H}_{2}$ molecules (Fig. 1g,h). To explain this observation, we analyze the Löwdin charges and find for $\mathrm{Ni}_{6}$ that adsorption of an $\mathrm{H}_{2}$ molecule shifts $\mathrm{Ni}_{6} 4 s$ spin-up states above the Fermi energy and Ni $3 d$ spin-down states below the Fermi energy, thereby decreasing the magnetic moment of the cluster. Moreover, a partial charge of 0.04 electrons is transferred to the $\mathrm{H}_{2}$ molecule, which is therefore elongated from $0.75 \AA$ to $0.89 \AA$. This dissociative $\mathrm{H}_{2}$ adsorption has been reported for $\mathrm{Ni}$ and other metal particles. ${ }^{33-36}$ Additional $\mathrm{H}_{2}$ molecules lead to similar effects. Löwdin charge analysis certifies that the magnetic moments of the $\mathrm{Ni}$ atoms with $\mathrm{H}_{2}$ molecules adsorbed are reduced (typically by $15 \%$ ) as compared to the other $\mathrm{Ni}$ atoms. $\mathrm{H}_{2}$ adsorption on $\mathrm{Ni}_{13}$ also shifts spin-up and spin-down states across the Fermi energy in opposite directions and the magnetic moment of the cluster decreases. The fact that this decrease is smaller than for $\mathrm{Ni}_{6}$ can be attributed to the fact that only the surface atoms participate in the $\mathrm{Ni}-\mathrm{H}$ bonding.

\subsection{Ni clusters on pristine graphene}

We next discuss results for the $\mathrm{Ni}$ clusters adsorbed on graphene $\left(\mathrm{Ni}_{x} / \mathrm{G}\right)$, with a binding energy of

$$
E_{b}=\frac{1}{x}\left(E(\mathrm{Y})+E\left(\mathrm{Ni}_{x}\right)-E\left(\mathrm{Ni}_{x}+\mathrm{Y}\right)\right)
$$

where $\mathrm{Y}$ is the graphene or GNM template. $\mathrm{Ni}_{6}$ shows slight deformations (Fig. 2a) with an average Ni-Ni distance of $2.35 \AA$. The average Ni-C distance is $2.09 \AA$, the binding energy per $\mathrm{Ni}$ atom is $0.16 \mathrm{eV}$, and the magnetic moment per $\mathrm{Ni}$ atom is $1.0 \mu_{\mathrm{B}}$. For $\mathrm{Ni}_{13}$ (Fig.

2b) we obtain an average Ni-Ni bond length of $2.41 \AA$, an average Ni-C distance of $2.07 \AA$, a binding energy per $\mathrm{Ni}$ atom of $0.20 \mathrm{eV}$, and a magnetic moment per Ni atom of $0.9 \mu_{\mathrm{B}}$. 
The deformation of the $\mathrm{Ni}_{13}$ cluster is not significantly affected by how it faces graphene; it ends up having triangular faces only. Löwdin charge analysis indicates that the average charge transfer from a $\mathrm{Ni}$ atom to graphene is 0.4 electrons for $\mathrm{Ni}_{6}$ and 0.6 electrons for $\mathrm{Ni}_{13}$, which explains the difference in the binding energy per $\mathrm{Ni}$ atom between the two clusters. The (partial, P) DOS (Fig. 2c,d) shows mainly Ni $3 d$ spin-down states at the Fermi energy and spin-up gaps of $0.25 \mathrm{eV}$ for $\mathrm{Ni}_{6} / \mathrm{G}$ and $0.70 \mathrm{eV}$ for $\mathrm{Ni}_{13} / \mathrm{G}$.

The relaxed structures after $\mathrm{H}_{2}$ adsorption (Fig. 2e,f) demonstrate that the maximum number of adsorbed $\mathrm{H}_{2}$ molecules is lower than in the case of the isolated clusters, which is due to the binding between some Ni atoms and graphene. The adsorption has no significant effect on the average Ni-C distance. Both systems can adsorb $8 \mathrm{H}_{2}$ molecules (Fig. 2g,h) with an average $\mathrm{H}-\mathrm{H}$ distance of $0.91 \AA$ and $0.84 \AA$ and an average $E_{\text {ad }}$ of $0.38 \mathrm{eV}$ and $0.50 \mathrm{eV}$ for $\mathrm{Ni}_{6} / \mathrm{G}$ and $\mathrm{Ni}_{13} / \mathrm{G}$, respectively. Because of the interaction with graphene, the magnetic moments are lower than those of the isolated clusters, with $\mathrm{Ni}_{6} / \mathrm{G}$ being completely depolarized at maximum $\mathrm{H}_{2}$ load. When more $\mathrm{H}_{2}$ molecules are adsorbed, again Ni spinup/down states are shifted above/below the Fermi energy, which decreases the magnetic moment.

\subsection{Ni clusters on GNMs}

We find that the Ni clusters bind weakly (binding energy per $\mathrm{Ni}$ atom: $0.08 \mathrm{eV}$ for $\mathrm{Ni}_{6}$ and $0.07 \mathrm{eV}$ for $\mathrm{Ni}_{13}$ ) to the O-GNM, which is reflected by average Ni-O distances of $2.10 \AA$ for $\mathrm{Ni}_{6}$ and $2.13 \AA$ for $\mathrm{Ni}_{13}$ (Fig. 3a,b). The average Ni-Ni distance is $2.31 \AA$ for $\mathrm{Ni}_{6}$ and $2.40 \AA$ for $\mathrm{Ni}_{13}$. We find at the Fermi energy mainly $\mathrm{Ni} 3 d$ spin-down states (Fig. 3c,d). Moreover, $6 \mathrm{H}_{2}$ molecules can be adsorbed on $\mathrm{Ni}_{6} / \mathrm{O}-\mathrm{GNM}$ and $8 \mathrm{H}_{2}$ molecules on $\mathrm{Ni}_{13} / \mathrm{O}-\mathrm{GNM}$ (Fig. 3e,f), with an average $\mathrm{H}-\mathrm{H}$ distance of $0.90 \AA$ and $0.85 \AA$ and an average $E_{\text {ad }}$ of $0.52 \mathrm{eV}$ and $0.49 \mathrm{eV}$, respectively. Again, the adsorption of $\mathrm{H}_{2}$ molecules reduces the magnetic moment (Fig. 3g,h). 
Figure 2: Optimized structures of (a) $\mathrm{Ni}_{6} / \mathrm{G}$ and (b) $\mathrm{Ni}_{13} / \mathrm{G}$, and (c,d) corresponding DOS/PDOS. Optimized structures of (e) $\mathrm{Ni}_{6} / \mathrm{G}$ and (f) $\mathrm{Ni}_{13} / \mathrm{G}$ with $\mathrm{H}_{2}$ molecules adsorbed, and corresponding (g) $E_{\text {ad }}$ and (h) $\mu$ per Ni atom as functions of $n$. 

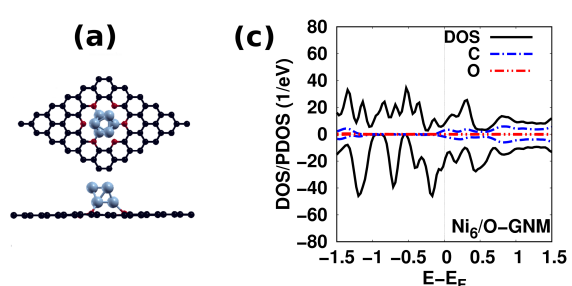

(b)
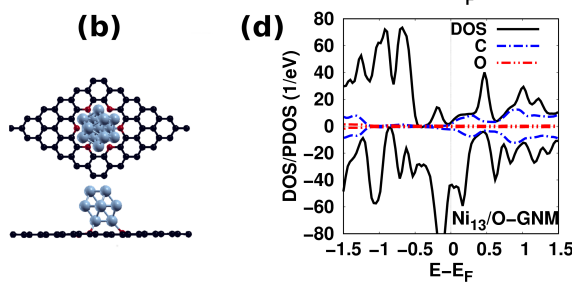

(e)

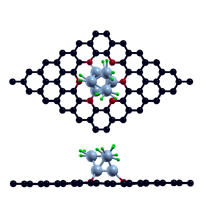

(g)

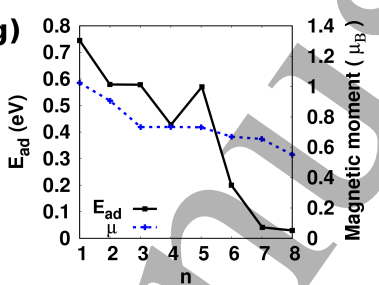

(f)

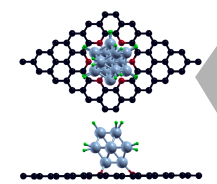

(h)

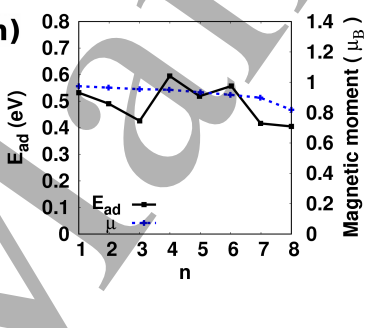

Figure 3: Optimized structures of (a) $\mathrm{Ni}_{6} / \mathrm{O}-\mathrm{GNM}$ and (b) $\mathrm{Ni}_{13} / \mathrm{O}-\mathrm{GNM}$, and (c,d) corresponding DOS/PDOS. Optimized structures of (e) $\mathrm{Ni}_{6} / \mathrm{O}-\mathrm{GNM}$ and (f) $\mathrm{Ni}_{13} / \mathrm{O}-\mathrm{GNM}$ with $\mathrm{H}_{2}$ molecules adsorbed, and corresponding (g) $E_{\mathrm{ad}}$ and (h) $\mu$ per Ni atom as functions of $n$.

Turning to the N-GNM, we first notice that both clusters form short bonds $(1.94 \AA$ for $\mathrm{Ni}_{6}$ and $1.97 \AA$ for $\mathrm{Ni}_{13}$ ) to the $\mathrm{N}$ atoms at the pore edge (Fig. 4a,b), because $\mathrm{N}$ has one valence electron more available than $\mathrm{O}$. The average $\mathrm{Ni}-\mathrm{Ni}$ distance is $2.31 \AA$ for $\mathrm{Ni}_{6}$ and $2.39 \AA$ for $\mathrm{Ni}_{13}$. We find at the Fermi energy that the $\mathrm{C} p_{z}$ states dominate in the spin-up channel and the Ni $3 d$ states in the spin-down channel (Fig. 4c,d). The Ni-N bonds at the pore edge are much stronger than the Ni-O bonds in the case of the O-GNM (binding energy per Ni atom: $1.42 \mathrm{eV}$ for $\mathrm{Ni}_{6}$ and $0.7 \mathrm{eV}$ for $\mathrm{Ni}_{13}$ ). Both starting configurations of the $\mathrm{Ni}_{13}$ cluster in the GNM pore give the same equilibrium structure. The cluster becomes slightly deformed having only triangular faces, different from the severe deformations occurring at 
unpassivated graphene vacancies. ${ }^{37}$ We obtain for $\mathrm{Ni}_{6}$ a hydrogen storage capacity of $6 \mathrm{H}_{2}$ molecules (Fig. 4e), with an average $\mathrm{H}-\mathrm{H}$ distance of $0.87 \AA$ and an average $E_{\text {ad }}$ of 0.36 $\mathrm{eV}$. As for $\mathrm{Ni}_{13}$ a smaller fraction of the cluster atoms is involved in the $\mathrm{Ni}-\mathrm{N}$ binding at the pore edge, a higher hydrogen storage capacity of $8 \mathrm{H}_{2}$ molecules is achieved (Fig. Af), with an average $\mathrm{H}-\mathrm{H}$ distance of $0.85 \AA$ and an average $E_{\text {ad }}$ of $0.45 \mathrm{eV}$. Similar to the other studied cases, the adsorption of $6 \mathrm{H}_{2}$ molecules reduces the magnetic moment (Fig. 4g,h). It turns out that the N-GNM offers the lowest mobility for the anchored Ni clusters (Fig. 5). However, the $\mathrm{H}_{2}$ storage capacity is low due to the fact that charge is consumed by the covalent bonds with the pore edge. This can be remedied by using large pore N-GNMs to host bigger $\mathrm{Ni}$ clusters and achieve $\mathrm{H}_{2}$ storage properties close to those of the isolated clusters. 


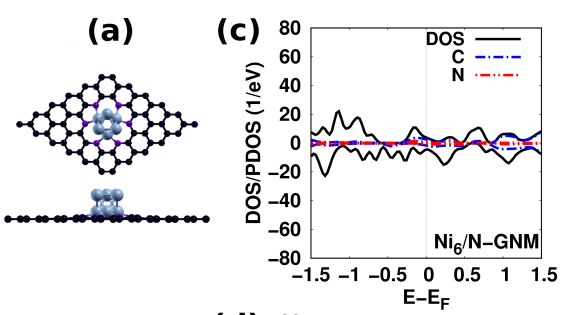

(b)

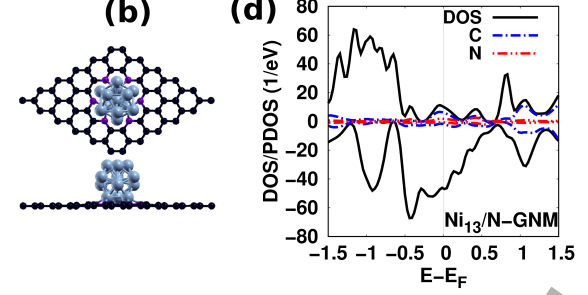

(e)
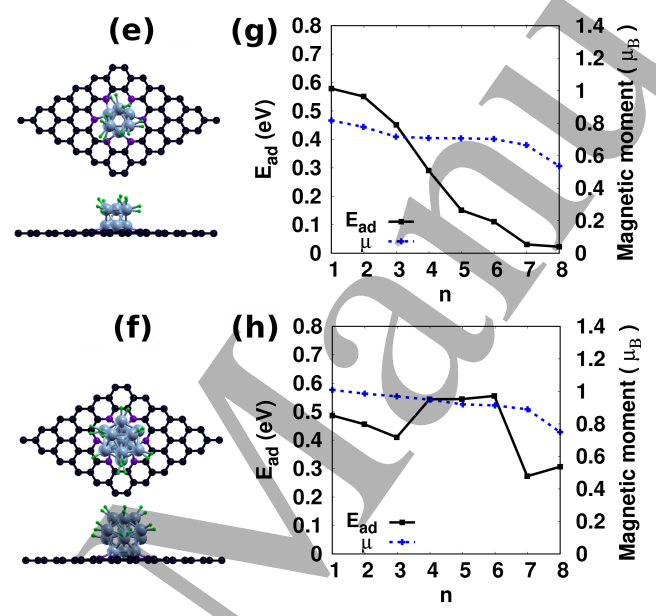

Figure 4: Optimized structures of (a) $\mathrm{Ni}_{6} / \mathrm{N}-\mathrm{GNM}$ and (b) $\mathrm{Ni}_{13} / \mathrm{N}-\mathrm{GNM}$, and (c,d) corresponding DOS/PDOS. Optimized structures of (e) $\mathrm{Ni}_{6} / \mathrm{N}-\mathrm{GNM}$ and (f) $\mathrm{Ni}_{13} / \mathrm{N}-\mathrm{GNM}$ with $\mathrm{H}_{2}$ molecules adsorbed, and corresponding (g) $E_{\text {ad }}$ and (h) $\mu$ per Ni atom as functions of $n$.

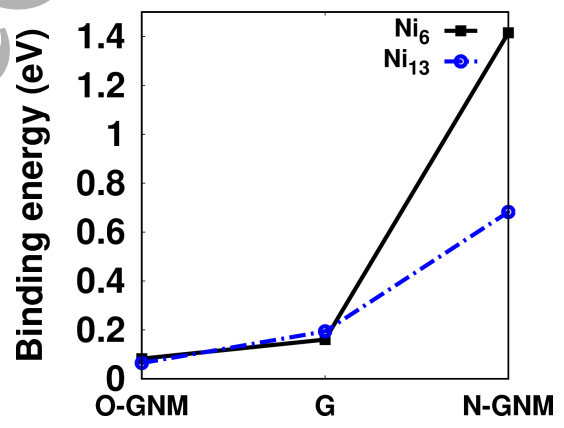

Figure 5: Binding energy per $\mathrm{Ni}$ atom of the Ni clusters on the different templates.

For $\mathrm{Ni}_{x} / \mathrm{G}$ the spin-up DOS is always gapped and the spin-down DOS always finite at the 
Fermi energy, which indicates that this system can be used as spin filter (because transport is permitted only for one spin component). As an analysis of the wavefunction demonstrates for the $\mathrm{Ni}_{13} / \mathrm{N}$-GNM that the Ni $3 d$ states (dominate at the Fermi energy the spin-down channel) are localized on the cluster while the $\mathrm{C} p_{z}$ states (dominate at the Fermi energy the spin-up channel) are delocalized, also this system can act as spin filter. When the pore density is decreased the spin-down states will slowly recover their graphene character and the spin filtering properties will be lost.

\section{Conclusion}

GNMs are porous graphene structures that are currently considered for applications in nanoelectronics, chemical separation, and molecular sensing. During or post fabrication, their pore edges can be selectively passivated to match the target application. Here, we use density functional theory to explore possible utilization for hydrogen storage as templates for anchoring Ni clusters on that $\mathrm{H}_{2}$ molecules can be adsorbed. We also study the magnetic properties. We find that the N-GNM is capable of firmly anchoring the Ni clusters through strong binding to the pore $\mathrm{N}$ atoms, while the O-GNM binds clusters only weakly. On the N-GNM the $\mathrm{Ni}_{6}$ and $\mathrm{Ni}_{13}$ clusters can host up to 6 and $8 \mathrm{H}_{2}$ molecules, respectively. The $\mathrm{H}_{2}$ storage properties of larger clusters are expected to approach those of Ni clusters on graphene, as the fraction of $\mathrm{Ni}$ atoms involved in the binding to the GNM decreases. We also find that for small numbers of adsorbed $\mathrm{H}_{2}$ molecules ( $n=2$ to 4 or 5 ) there is some variation in the adsorption energy per molecule $(\sim 0.1 \mathrm{eV})$ due to the low density of $\mathrm{H}_{2}$ molecules. This variation decreases as more $\mathrm{H}_{2}$ molecules are added to the Ni clusters. For larger clusters less variation in the adsorption energies is expected, as the molecules are more homogeneously distributed over the cluster.

We observe half-metallic properties for $\mathrm{Ni}_{x} / \mathrm{G}$, opening potential for spin filtering applications. On the other hand, $\mathrm{Ni}_{13} / \mathrm{N}$-GNM shows at the Fermi energy a finite DOS for both 


\section{Acknowledgment}

The authors would like to acknowledge the use of the resources of the Supercomputing Laboratory at KAUST, and the resources and technical services provided by the Scientific and High Performance Computing Center at Imam Abdulrahman Bin Faisal University, Dammam, Saudi Arabia. M. Fadlallah would also like to thank Ulrich Eckern for fruitful discussions.

\section{References}

(1) Solomon, S.; Plattner, G.-K.; Knutti, R.; Friedlingstein, P. Irreversible climate change due to carbon dioxide emissions. Proceedings of the National Academy of Sciences 2009, 1704-1709.

(2) Jain, I. Hydrogen the fuel for 21st century. International Journal of Hydrogen Energy 2009, 34, 7368-7378.

(3) Chen, P.; Zhu, M, Recent progress in hydrogen storage. Materials Today 2008, 11, $36-43$.

(4) Durbin, D.; Malardier-Jugroot, C. Review of hydrogen storage techniques for on board vehicle applications. International Journal of Hydrogen Energy 2013, 38, 14595-14617.

(5) Han, S. S.; Mendoza-Cortes, J. L.; Goddard III, W. A. Recent advances on simulation and theory of hydrogen storage in metal-organic frameworks and covalent organic frameworks. Chemical Society Reviews 2009, 38, 1460-1476. 
(6) Zheng, J.; Liu, X.; Xu, P.; Liu, P.; Zhao, Y.; Yang, J. Development of high pressure gaseous hydrogen storage technologies. International Journal of Hydrogen Energy 2012, 37, 1048-1057.

(7) Broom, D. P. et al. Outlook and challenges for hydrogen storage in nanoporous materials. Applied Physics A 2016, 122, 151.

(8) Navlani-García, M.; Mori, K.; Kuwahara, Y.; Yamashita, H. Recent strategies targeting efficient hydrogen production from chemical hydrogen storage materials over carbonsupported catalysts. NPG Asia Materials 2018, 10, 277-292.

(9) Reyhani, A.; Mortazavi, S.; Mirershadi, S.; Moshfegh, A., Parvin, P.; Golikand, A. N. Hydrogen storage in decorated multiwalled carbon nanotubes by $\mathrm{Ca}, \mathrm{Co}, \mathrm{Fe}, \mathrm{Ni}$, and Pd nanoparticles under ambient conditions. The Journal of Physical Chemistry C 2011, $115,6994-7001$.

(10) Seenithurai, S.; Pandyan, R. K.; Kumar, S. V.; Saranya, C.; Mahendran, M. Lidecorated double vacancy graphene for hydrogen storage application: A first principles study. International Journal of Hydrogen Energy 2014, 39, 11016-11026.

(11) Sigal, A.; Rojas, M.; Leiva, E. Interferents for hydrogen storage on a graphene sheet decorated with nickel: A DFT study. International Journal of Hydrogen Energy 2011, $36,3537-3546$.

(12) Lee, H.; Ihm, J.; Cohen, M. L.; Louie, S. G. Calcium-decorated graphene-based nanostructures for hydrogen/storage. Nano Letters 2010, 10, 793-798.

(13) Burress, J.; Kraus, M.; Beckner, M.; Cepel, R.; Suppes, G.; Wexler, C.; Pfeifer, P. Hydrogen storage in engineered carbon nanospaces. Nanotechnology 2009, 20, 204026.

(14) Reunchan, P.; Jhi, S.-H. Metal-dispersed porous graphene for hydrogen storage. Applied Physics Letters 2011, 98, 093103. 
(15) Li, S.; Zhao, H.-M.; Jena, P. Ti-doped nano-porous graphene: A material for hydrogen storage and sensor. Frontiers of Physics 2011, 6, 204-208.

(16) Ouyang, F.; Peng, S.; Liu, Z.; Liu, Z. Bandgap opening in graphene antidot lattices: The missing half. ACS Nano 2011, 5, 4023-4030.

(17) Petersen, R.; Pedersen, T. G.; Jauho, A.-P. Clar sextet analysis of triangular, rectangular, and honeycomb graphene antidot lattices. ACS Nano 2010, 5, 523-529.

(18) Eldeeb, M. S.; Fadlallah, M. M.; Martyna, G. J.; Maarouf, A. A. Doping of large-pore crown graphene nanomesh. Carbon 2018, 133, 369-378.

(19) Chen, H.; Zhang, S.; Jiang, W.; Zhang, C.; Guo, H.; Liu, Z.; Wang, Z.; Liu, F.; Niu, X. Prediction of two-dimensional nodal-line semimetals in a carbon nitride covalent network. Journal of Materials Chemistry A 2018, 6, 11252-11259.

(20) Maarouf, A. A.; Nistor, R. A.; Afzali-Ardakani, A.; Kuroda, M. A.; Newns, D. M.; Martyna, G. J. Crown graphene nanomeshes: Highly stable chelation-doped semiconducting materials. Journal of Chemical Theory and Computation 2013, 9, 2398-2403.

(21) Abou-Kandil, A.; Maarouf, A.; Martyna, G.; Mohamed, H.; Newns, D. Doped, passivated graphene nanomesh, method of making the doped, passivated graphene nanomesh, and semiconductor device including the doped, passivated graphene nanomesh. 2014; US Patent 8,900,538.

(22) Yang, H.-X.; Chshiev, M.; Boukhvalov, D. W.; Waintal, X.; Roche, S. Inducing and optimizing magnetism in graphene nanomeshes. Physical Review B 2011, 84, 214404.

(23) Afzali, A.; Maarouf, A.; Martyna, G. Controlled assembly of charged nanoparticles using functionalized graphene nanomesh. 2014; US Patent 8,835,686.

(24) Bai, J.; Zhong, X.; Jiang, S.; Huang, Y.; Duan, X. Graphene nanomesh. Nature nanotechnology 2010, 5, 190-194. 
(25) Konig, M.; Ruhl, G.; Batke, J.-M.; Lemme, M. C. Self-organized growth of graphene nanomesh with increased gas sensitivity. Nanoscale 2016, 8, 15490-15496.

(26) Paul, R. K.; Badhulika, S.; Saucedo, N. M.; Mulchandani, A. Graphene nanomesh as highly sensitive chemiresistor gas sensor. Analytical Chemistry 2012, 84, 8171-8178.

(27) Afzali, A.; Maarouf, A.; Martyna, G. Graphene nanomesh based charge sensor. 2014; US Patent 9,102,540.

(28) Giannozzi, P. et al. QUANTUM ESPRESSO: A modular and open-source software project for quantum simulations of materials. Journal of Physics: Condensed Matter 2009, 21, 395502 .

(29) Monkhorst, H. J.; Pack, J. D. Special points for Brillouin-zone integrations. Physical Review B 1976, 13, 5188-5192.

(30) Buschow, K. H. J. Handbook of magnetic/materials; Elsevier: Amsterdam, 2003; Vol. 15 .

(31) Reddy, B.; Nayak, S.; Khanna, S.; Rao, B.; Jena, P. Physics of nickel clusters. 2. Electronic structure and magnetic properties. The Journal of Physical Chemistry A 1998, 102, 1748-1759.

(32) Rodrguez-Kessler, P. L.; Rodrguez-Domnguez, A. R. Stability of Ni clusters and the adsorption of $\mathrm{CH}_{4}$ : First-principles calculations. The Journal of Physical Chemistry C $2015,119,12378-12384$.

(33) Ambrusi, R. E.; Pronsato, M. E. DFT study of Rh and Ti dimers decorating N-doped pyridinic and pyrrolic graphene for molecular and dissociative hydrogen adsorption. Applied Surface Science 2019, 464, 243-254.

(34) López-Corral, I.; Piriz, S.; Faccio, R.; Juan, A.; Avena, M. A van der Waals DFT study 
of $\mathrm{PtH}_{2}$ systems absorbed on pristine and defective graphene. Applied Surface Science 2016, 382, 80-87.

(35) Ambrusi, R. E.; Luna, C. R.; Juan, A.; Pronsato, M. E. DFT study of Rh-decorated pristine, B-doped and vacancy defected graphene for hydrogen adsorption. RSC Advances 2016, 6, 83926-83941.

(36) López-Corral, I.; Germán, E.; Juan, A.; Volpe, M.; Brizuela, G. Hydrogen adsorption on palladium dimer decorated graphene: A bonding study. International Journal of Hydrogen Energy 2012, 37, 6653-6665.

(37) Gao, W.; Mueller, J. E.; Anton, J.; Jiang, Q.; Jacob, T. Nickel cluster growth on defect sites of graphene: A computational study. Angewandte Chemie - International Edition 2013, 52, 14237-14241. 